Nonlin. Processes Geophys., 25, 201-205, 2018

https://doi.org/10.5194/npg-25-201-2018

(C) Author(s) 2018. This work is distributed under

the Creative Commons Attribution 4.0 License.

\title{
Brief communication: A nonlinear self-similar solution to barotropic flow over varying topography
}

\author{
Ruy Ibanez ${ }^{1}$, Joseph Kuehl ${ }^{2}$, Kalyan Shrestha ${ }^{3}$, and William Anderson ${ }^{3}$ \\ ${ }^{1}$ Mechanical Engineering Department, University of Rochester, Rochester, NY 14627, USA \\ ${ }^{2}$ Mechanical Engineering Department, University of Delaware, Newark, DE 19716, USA \\ ${ }^{3}$ Mechanical Engineering Department, University of Texas Dallas, Dallas, TX 75080, USA
}

Correspondence: Joseph Kuehl (jkuehl@udel.edu)

Received: 1 November 2017 - Discussion started: 14 November 2017

Revised: 25 January 2018 - Accepted: 31 January 2018 - Published: 6 March 2018

\begin{abstract}
Beginning from the shallow water equations (SWEs), a nonlinear self-similar analytic solution is derived for barotropic flow over varying topography. We study conditions relevant to the ocean slope where the flow is dominated by Earth's rotation and topography. The solution is found to extend the topographic $\beta$-plume solution of Kuehl (2014) in two ways. (1) The solution is valid for intensifying jets. (2) The influence of nonlinear advection is included. The SWEs are scaled to the case of a topographically controlled jet, and then solved by introducing a similarity variable, $\eta=c x^{n_{x}} y^{n_{y}}$. The nonlinear solution, valid for topographies $h=h_{0}-\alpha x y^{3}$, takes the form of the Lambert $W$-function for pseudo velocity. The linear solution, valid for topographies $h=h_{0}-\alpha x y^{-\gamma}$, takes the form of the error function for transport. Kuehl's results considered the case $-1 \leq \gamma<1$ which admits expanding jets, while the new result considers the case $\gamma<-1$ which admits intensifying jets and a nonlinear case with $\gamma=-3$.
\end{abstract}

\section{Introduction}

Slope topography represents both a barrier to large-scale geophysical fluid transport as well as an important location of mesoscale feature generation. Standard quasi-geostrophic theory (Pedlosky, 1987) indicates that large-scale circulation features act in such a way as to conserve their potential vorticity, leading to the standard result of flow along (as opposed to across) topographic contours. Thus, slope topography creates a barrier between the open and coastal oceans, often inhibiting the transport of nutrient-rich waters into the coastal zone and at the same time trapping pollutants in the coastal zone.

As both numerical and observational approaches have limitations with respect to modeling the slope region, the objective of this brief communication is to provide an analytic framework for flow along slope topographies. Such a framework will serve as an idealized backbone upon which observational, numerical, experimental and further theoretical work can build and provide a point of comparison for better interpretation of the respective dynamics. In particular, the results presented have implications for cross-topography exchange and provide significant insight into the coupling between the slope bottom boundary layer and interior water column dynamics.

\section{Problem formulation}

The problem formulation considered in this work follows that of Sansón and van Heijst (2002), Kuehl (2014) and Kuehl and Sheremet (2014). A rotating, single fluid layer is considered which flows along a sloping bottom topography (i.e., along slope barotropic flow). The momentum equations and continuity Eq. (1) for this situation are

$$
\begin{aligned}
& u_{t}-(f+\omega) v=-(p+e)_{x}+v \nabla^{2} u, \\
& v_{t}+(f+\omega) u=-(p+e)_{y}+v \nabla^{2} v, \\
& h_{t}+(h u)_{x}+(h v)_{y}+\nabla \cdot \Pi_{\mathrm{E}}=0,
\end{aligned}
$$

(Pedlosky, 1987; Cushman-Roisin, 1994, provide the scaling which leads to these equations) where $u, v$ are the across and along slope flow velocities, respectively, $h$ is the fluid 
depth of the at rest state, $p$ is the pressure anomaly divided by water density $(\rho), e=\left(u^{2}+v^{2}\right) / 2$ is kinetic energy per unit mass, $v$ is the viscosity and $f$ is the Coriolis parameter. The effect of the viscous bottom boundary layer is accounted for by a small correction term $\Pi_{\mathrm{E}}=\frac{1}{2} h_{\mathrm{E}} \boldsymbol{k} \times \boldsymbol{u}$, the Ekman flux. Its divergence, $\nabla \cdot\left(\Pi_{\mathrm{E}}\right)=-\frac{1}{2} h_{\mathrm{E}} \omega$, represents first-order Ekman suction at a solid boundary with Ekman layer depth, $h_{\mathrm{E}}=\sqrt{2 v / f}$. Taking the curl of the momentum equations, defining the vorticity as $\omega=v_{x}-u_{y}$, defining an interior transport function $\psi$ through $h \boldsymbol{u}=\hat{k} \times \nabla \psi+\nabla \phi$ (where $\nabla^{2} \phi=-\nabla \cdot \Pi_{\mathrm{E}}=\frac{1}{2} h_{\mathrm{E}} \omega$ represents Ekman divergence), and simplifying by letting $q=\frac{f+\omega}{h}$, gives us the vorticity-transport Eq. (2),

$\omega_{t}+J(\psi, q)=v \nabla^{2} \omega-\frac{h_{\mathrm{E}}}{2} q \omega$.

The divergent component caused by the Ekman suction is small $\left(\phi / \psi=h_{\mathrm{E}} / h=O\left(10^{-2}\right)\right.$, so $\phi$ can be neglected in the vorticity advection terms. It is standard to expand the Jacobian $\left[J(\psi, q)=\psi_{x} q_{y}-\psi_{y} q_{x}\right], J(\psi, q)=\frac{1}{h} J(\psi, \omega)-$ $\frac{f}{h^{2}} J(\psi, \eta)+\frac{\beta^{(x)}}{h} \psi_{y}-\frac{\beta^{(y)}}{h} \psi_{x}$, where $\beta^{(x)}=\left(h_{x} f\right) / h$ and $\beta^{(y)}=\left(h_{y} f\right) / h$ are the average topographic beta-effects and $\eta$ is a small free-surface displacement and thus the total water column depth is $h+\eta$.

Kuehl (2014) provides a scaling analysis which justified Ekman dissipation being the dominant dissipative term and relative vorticity being dominated by cross-stream shear, $\omega \approx \frac{1}{h} \psi_{x x}$. These assumptions are valid for flows which exhibit scale separation between the along and cross flow (topography) directions and are thus valid for flow along the oceanic slope. These assumptions, along with the steady flow assumption, truncate a Taylor expansion in $\frac{1}{h}$ at leading order (neglecting terms of $O\left(\frac{1}{h^{2}}\right)$ ) and assume $f \gg \omega$ are again made and result in a leading-order governing equation of the form

$\psi_{x} \psi_{x x y}-\psi_{y} \psi_{x x x}+f h_{x} \psi_{y}-f h_{y} \psi_{x}=-\frac{f h_{\mathrm{E}}}{2} \psi_{x x}$.

This equation (with appropriate boundary conditions) describes the linear and first-order nonlinear dynamics of a barotropic flow along the oceanic slope. It is upon this equation that several analytic solutions will be presented.

\section{Linear solutions}

\subsection{Expanding jet}

Kuehl (2014) considered the linear case of Eq. (3),

$f h_{x} \psi_{y}-f h_{y} \psi_{x}=-\frac{f h_{\mathrm{E}}}{2} \psi_{x x}$.

Noting its similarity to the heat equation, which has been pointed out by others (in particular Csanady, 1978), Kuehl attempted to find a similarity solution. The solution derivation will be sketched through here for completeness (details in Kuehl, 2014). Assuming

- topography of the form $h=h_{0}-\alpha x y^{-\gamma}$,

- similarity variable $\zeta=x(k y)^{n}$,

- boundary conditions $\psi(-\infty, y)=0$ and $\psi(\infty, y)=Q$,

- initial condition $\psi(x, 0)=Q \operatorname{sgn}(x)$,

Eq. (4) reduces to $-2 \zeta g^{\prime}=g^{\prime \prime}$, where $g=\psi / Q$, with conditions $n=-\frac{1+\gamma}{2}$ and $k=\left[\frac{\alpha}{2 h_{\mathrm{E}}}(1-\gamma)\right]^{\frac{1}{2 n}}$. This equation has a well-known solution, $\psi=Q\left[\frac{\operatorname{erf}(\zeta)+1}{2}\right]$, and parameters $\alpha$ and $\gamma$ may be set to mimic the desired topography. The "topographic $\beta$-plume" solution is valid in the parameter range $-1 \leq \gamma<1$. For the solution to be real, we must have $\gamma<1$ and, for $\gamma<-1$, the jet would be compressing, which does not satisfy the initial conditions. Physically, the Ekman pumping in the bottom boundary layer relaxes the topographic vorticity control, allowing the jet to spread across isobaths.

\subsection{Compressing jet}

In nature, compressing (or intensifying) jets are often observed and an analysis of ocean slope topography finds many locations where $\gamma<-1$ is relevant (Ibanez, 2016). To extend the above result to the case of compressing jets, the initial condition used above must be revisited. Similarity solutions require one point of reference to tether the solution. It is most common to place this singularity at the origin, as is done above and in many other classical cases such as the Blasius boundary layer (Blasius, 1908; Rogers, 1992). However, in the present case, we choose to relocate the singularity to $y=\infty$. Upon relocation, the solution given above is still valid, but the domain of physical relevance of the solution has a slightly altered interpretation.

For the expanding jet case, the analytical solution is valid over the domain $y=[0: \infty]$. However, the physical relevance of the solution demands the neglect of the region near $y=0$, due to the singularity, as well as the region near $y=\infty$, as this region violates the across and along jet scale separation assumption, though the interior solution is indeed a physically relevant description of geophysical systems. For the compressing jet case, the situation is simply reversed. In this case, the analytical solution is still valid over the domain $y=[0: \infty]$. However, the physical relevance of the solution demands the neglect of the region near $y=0$, as this region violates the across and along jet scale separation assumption, and the region near $y=\infty$, due to the singularity, but again the interior solution is a physically relevant description of geophysical systems. The region of applicability is ultimately governed by the assumption $\omega \approx \frac{1}{h} \psi_{x x}$ (i.e., $\psi_{x x} \gg \psi_{y y}$ ), 
which is reasonable but should be checked in each particular application. Thus, we have adopted the terminology that expanding jets are those with a singularity at the upstream source region $(y=0)$ and compressing jets as those with the singularity at the downstream exit region $(y=\infty)$.

\section{Nonlinear solution}

Motivated by the success and utility of the linear solutions provided above, we seek a similarity solution for the nonlinear case (Eq. 3). Again, consider the normalized transport function, $g=\frac{\psi}{Q}$, and introduce a similarity variable of the form $\eta=c x^{n_{x}} y^{n_{y}}$, where $c, n_{x}, n_{y}$ are constants. Note that from this point on $\eta$ will refer to the similarity variable and not surface displacement. The relevant derivatives take the forms

$$
\begin{aligned}
g_{y} & =g^{\prime} \frac{\partial \eta}{\partial y}=n_{y} c x^{n_{x}} y^{n_{y}-1} g^{\prime}, \\
g_{x} & =g^{\prime} \frac{\partial \eta}{\partial x}=n_{x} c x^{n_{x}-1} y^{n_{y}} g^{\prime}, \\
g_{x x} & =\frac{\partial}{\partial x}\left[g^{\prime} \frac{\partial \eta}{\partial x}\right]=g^{\prime \prime}\left(\frac{\partial \eta}{\partial x}\right)^{2}+g^{\prime} \frac{\partial^{2} \eta}{\partial x^{2}} \\
& =n_{x}^{2} c^{2} x^{2\left(n_{x}-1\right)} y^{2 n_{y}} g^{\prime \prime}+n_{x}\left(n_{x}-1\right) c x^{n_{x}-2} y^{n_{y}} g^{\prime}, \\
g_{x x x} & =g^{\prime \prime \prime}\left(n_{x}^{3} c^{3} x^{3\left(n_{x}-1\right)} y^{3 n_{y}}\right) \\
& +3 g^{\prime \prime}\left(n_{x} c x^{n_{x}-1} y^{n_{y}}\right)\left(n_{x}\left(n_{x}-1\right) c x^{n_{x}-2} y^{n_{y}}\right) \\
& +g^{\prime}\left(n_{x}\left(n_{x}-1\right)\left(n_{x}-2\right) c x^{n_{x}-3} y^{n_{y}}\right), \\
g_{x x y} & =g^{\prime \prime \prime}\left(n_{y} c x^{n_{x}} y^{n_{y}-1}\right)\left(c^{2} n_{x}^{2} x^{2\left(n_{x}-1\right)} y^{2 n_{y}}\right) \\
& +g^{\prime \prime}\left(2 n_{x} c x^{n_{x}-1} y^{n_{y}}\right)\left(n_{x} n_{y} c x^{n_{x}-1} y^{n_{y}-1}\right) \\
& +g^{\prime \prime}\left(n_{y} c x^{n_{x}} y^{n_{y}-1}\right)\left(n_{x}\left(n_{x}-1\right) x^{n_{x}-2} y^{n_{y}}\right) \\
& +g^{\prime}\left(c n_{x}\left(n_{x}-1\right) n_{y} x^{n_{x}-2} y^{n_{y}-1}\right) .
\end{aligned}
$$

In this work, we are interested in straight slope topographies. Upon setting $n_{x}=1$, it is seen that the nonlinear terms simplify significantly. Specifically, all $g^{\prime} g^{\prime}$ terms are set to zero. Also, it is found that the $g^{\prime} g^{\prime \prime \prime}$ terms cancel. Thus, the only remaining nonlinear term is the $g^{\prime} g^{\prime \prime}$ term, which in Eq. (3) takes the form $Q^{2} g^{\prime} g^{\prime \prime} c^{3} n_{y} y^{3 n_{y}-1}$. Ultimately, Eq. (3) becomes

$$
\begin{aligned}
& \underbrace{Q g^{\prime}\left[h_{x} f c n_{y} x y^{n_{y}-1}-h_{y} f c y^{n_{y}}\right]}_{1} \\
& +\underbrace{Q g^{\prime \prime}\left[\frac{f h_{e}}{2} c^{2} y^{2 n_{y}}\right]}_{2}+\underbrace{Q^{2} g^{\prime} g^{\prime \prime}\left[c^{3} n_{y} y^{3 n_{y}-1}\right]}_{3}=0 .
\end{aligned}
$$

It is now convenient to address the $y$ dependences of the coefficients in terms 2 and 3 of Eq. (5). We require the $y$ dependency of terms 2 and 3 to balance, i.e., $2 n_{y}=3 n_{y}-1$, which gives the condition $n_{y}=1$. Thus, the similarity variable has the form $\eta=c x y$. Apply this condition, and upon division by the coefficient of term 2 , this yields

$$
\underbrace{\frac{2}{h_{e} c} g^{\prime}\left[h_{x} x y^{-2}-h_{y} y^{-1}\right]}_{1}+\underbrace{g^{\prime \prime}}_{2}+\underbrace{\frac{2 Q c}{f h_{e}} g^{\prime} g^{\prime \prime}}_{3}=0 .
$$

Next, the bracketed portion of Eq. (6) in term 1 is considered. Recall that $h=h_{0}-\alpha x y^{-\gamma}, h_{x}=-\alpha y^{-\gamma}$ and $h_{y}=$ $\alpha \gamma x y^{-\gamma-1}$. We anticipate that the $x$ must be absorbed into an $\eta$ term, so the bracketed terms become

$-\frac{\alpha}{c} \eta y^{-\gamma-3}(1+\gamma)$.

The $y$ dependence is removed with the condition $\gamma=-3$ and the terms in Eq. (7) reduce to $2 \frac{\alpha}{c} \eta$. It is then found that Eq. (6) reduces to

$$
\frac{4 \alpha}{h_{e} c^{2}} \eta g^{\prime}+g^{\prime \prime}+\frac{2 Q c}{f h_{e}} g^{\prime} g^{\prime \prime}=0
$$

Note that, as expected, the limit of Eq. (8), as $Q \rightarrow 0$, recovers the linear solutions provided above with $\frac{4 \alpha}{h_{e} c^{2}}=2$.

Thus, for topography of the form $h=h_{0}-\alpha x y^{3}$ and a similarity variable of the form $\eta=c x y$, the nonlinear PDE, Eq. (3), reduces to a nonlinear ODE of the form

$\eta g^{\prime}=-\left(K_{1}+K_{2} g^{\prime}\right) g^{\prime \prime}$,

with $K_{1}=\frac{h_{e} c^{2}}{4 \alpha}$ and $K_{2}=\frac{Q c^{3}}{2 f \alpha}$.

Equation (9) can be solved for $g^{\prime}$ by using separation of variables. Let $g^{\prime}(\eta)=u(\eta)$ (the "pseudo velocity") so $\eta u=$ $-\left(K_{1}+K_{2} u\right) \frac{\mathrm{d} u}{\mathrm{~d} \eta}$ or $\eta d \eta=-\left(K_{1}+K_{2} u\right) \frac{\mathrm{d} u}{u}$. Integrating both sides yields

$\frac{\eta^{2}}{2}+m=-\left(K_{1} \ln u+K_{2} u\right)$,

where $m$ is an integration constant related to the total transport.

It is possible to solve Eq. (10) for $u$, by using the Lambert $W$-function $(W)$,

$u(\eta)=\frac{K_{1} W\left(\frac{K_{2}}{K_{1}} e^{-\frac{2 m+\eta^{2}}{2 K_{1}}}\right)}{K_{2}}$.

The integral of $u(\eta)$ is the analytic solution to the normalized transport equation, whose boundary conditions are $g(-\infty, y)=0, g(\infty, y)=1$ and $g(x, \infty)=Q \operatorname{sgn}(x)$. However, the solution to the derivative of the transport function (pseudo velocity, $u$ ) is sufficient to calculate the flow field, as $\psi_{x}=Q g^{\prime}(\eta) \frac{\mathrm{d} \eta}{\partial x}$ and $\psi_{y}=Q g^{\prime}(\eta) \frac{\mathrm{d} \eta}{\partial y}$. 

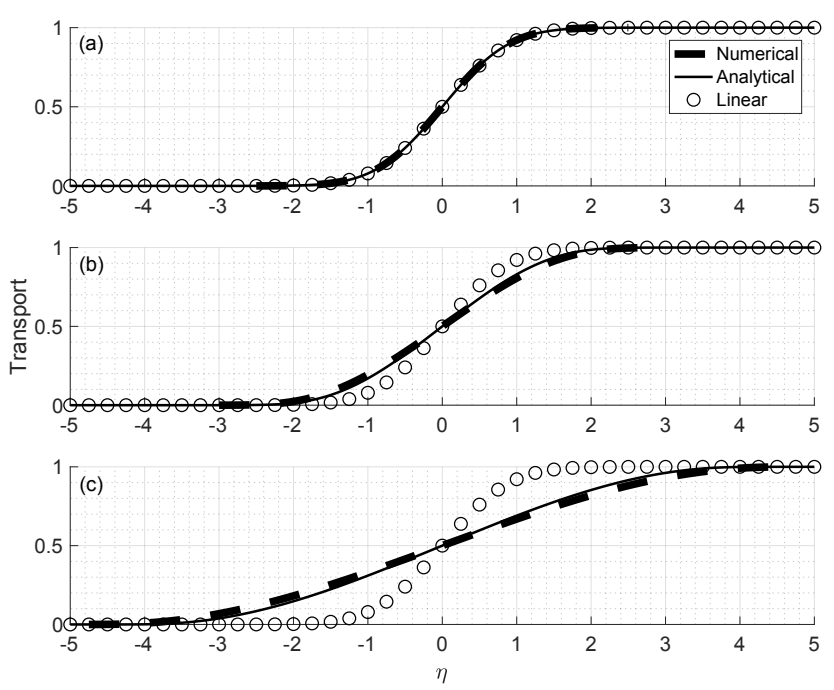

Figure 1. Comparison between linear (open circles), nonlinear numerical (thick dashed) and nonlinear analytic (solid lines) normalized transport functions. Plotted is the ratio $K_{2} / K_{1}$ (nonlinear coefficient over linear coefficient) of 0.001 (a), 10 (b) and 100 (c) with $K_{1}=0.5$.

\section{Calculation}

It can be seen that $m$ is related to total transport by taking the analytic limit of Eq. (11) as $K_{2} \rightarrow 0$ (which is an error function) and evaluating the transport boundary conditions. To complete the analytic solution in the nonlinear case, Eq. (11) can be integrated and an iterative method can be employed to determine $m$ based on the transport boundary condition. Alternatively, Eq. (9) can be solved numerically. A fourth-order Runge-Kutta method coupled with a shooting algorithm was applied to iteratively meet the total transport boundary condition. It should be noted that the iterative numerical approach is based on a very small and sensitive velocity boundary condition, which cannot be taken at $-\infty$ but must be approximated at a small finite value. In the linear and moderate nonlinear regimes, the numerical and analytical solutions show good agreement (Fig. 1). However, as nonlinearity increases, the velocity boundary condition becomes extremely sensitive and difficult to iterate on. Thus, the great advantage of an analytical solution is that it is easily applicable at any amplitude.

\section{Discussion}

The solutions presented above are relevant to barotropic, along slope flow over generic topographies of the form $h=$ $h_{0}-\alpha x y^{-\gamma}$. For the linear solution cases, the Ekman pumping relaxes the topographic vorticity control via the bottom boundary layer. When $-1 \leq \gamma<1$, the Ekman pumping out paces the topographic control and an expanding topographic $\beta$-plume solution is found. This represents cross-topographic transport due solely to bottom boundary layer processes. When $\gamma<-1$, the Ekman pumping is not able to overcome the topographic influence and a compressing topographic $\beta$ plume solution is found. Such compressing solutions result in intense currents, which may be subject to instability. For the special case, $h=h_{0}-\alpha x y^{3}$, a nonlinear solution is found. As seen in Fig. 1, the nonlinear solution broadens compared to the linear solution. At first this may seem to be a contradiction; however, one must remember that in this case the topographic slope is rapidly increasing, with the influence to compress the jet. The influence of the nonlinear terms is to resist this compression. This is consistent with the expected tendency of flow inertia. The details of this nonlinear tendency are then relevant to the onset of barotropic instability (or other forms of instability, analysis of which is ongoing work). Note also that the nonlinear solution limits to the linear solution (both analytically and numerically), as it must.

Code and data availability. This is an analytical paper: the codes described are standard and easily reproduced from explanations provided in the text.

Author contributions. Preparation of this paper was led by RI and JK; however, the ideas contained herein are the result of numerous discussions between all the authors listed.

Competing interests. The authors declare that they have no conflict of interest.

Acknowledgements. This work was supported by the Texas General Land Office, Oil Spill Program (program manager: Steve Buschang) under TGLO contract no. 16-019-0009283 and the National Science Foundation, Physical Oceanography Program (program manager: Eric Itsweire) under grant no. 1823452.

Edited by: Juan Restrepo

Reviewed by: two anonymous referees

\section{References}

Blasius, H.: Grenzschichten in Flüssigkeiten mit kleiner Reibung, Z. Angew. Math. Phys., 56, 1-37, 1908.

Csanady, G. T.: The arrested topographic wave, J. Phys. Oceanogr., 8, 47-62, 1978.

Cushman-Roisin, B.: Introduction to Geophysical Fluid Dynamics, 320 pp., Prentice-Hall Inc., Englewood Cliffs, New Jersey, 1994.

Ibanez, R.: Analytic Modeling and Applications of Barotropic Flows over Sloping Topographies Using Similarity Solutions, Masters Thesis, Baylor University, 2016.

Kuehl, J. J.: An analytic solution for barotropic flow along a variable slope topography, Geophys. Res. Lett., 41, 7591-7594, https://doi.org/10.1002/2014GL061188, 2014. 
Kuehl, J. J. and Sheremet, V. A.: Two-layer gap-leaping oceanic boundary currents: experimental investigation, J. Fluid Mech., 740, 97-113, 2014.

Lozier, M. S. and Reed, M.: The influence of topography on the stability of shelfbreak fronts, J. Phys. Oceanogr., 35, 1023-1036, 2005.

Pedlosky, J.: Geophysical Fluid Dynamics, Springer, New York, 1987.
Rogers, D. F.: Laminar Flow Analysis, Cambridge University Press, New York, 1992.

Sansón, L. and Van Heijst, G.: Ekman effects in a rotating flow over bottom topography, Journal of Fluid Mechanics, 471, 239-255, https://doi.org/10.1017/S0022112002002239, 2002. 